\title{
DISTRIBUTED VIDEO CODING: STATUS, CHALLENGES AND OUTLOOK
}

\author{
Frédéric Dufaux \\ Multimedia Signal Processing Group, Ecole Polytechnique Fédérale de Lausanne (EPFL) \\ CH-1015 Lausanne, Switzerland \\ Email: frederic.dufaux@epfl.ch
}

\begin{abstract}
This presentation surveys the current status of research activities on Distributed Video Coding (DVC). In particular, we review some recent contributions, identify challenges ahead, and outline perspectives.
\end{abstract}

Keywords - Distributed Video Coding, coding efficiency, complexity, error resilience, scalability, multiview

\section{INTRODUCTION}

Fast and efficient access to information, including multimedia information, is paramount in nowadays information society. Tremendous advances in computer and communication technologies have led to the ubiquitous use of digital media content and the successful deployment of new products and services. However, digital video is still demanding in terms of processing power, bandwidth and storage capacity. This digital revolution has only been possible thanks to the rapid and remarkable progresses in video coding technologies. In addition, standardization efforts in MPEG and ITU-T have played a key role in this endeavor.

Most past research activities on video coding has relied on the two principles of predictive and transform coding. All present MPEG and ITU-T video coding standards are based on motion compensated block-based Discrete Cosine Transform (DCT) hybrid design. The state-of-the-art H.264/Advanced Video Coding (AVC) standard is the latest installment in this series.

Distributed Source Coding (DSC) is a new coding paradigm which has appeared a few years ago. It based on the Slepian-Wolf [1] and Wyner-Ziv [2] Information Theory theorems from the 70's. The Slepian-Wolf theorem considers the lossless coding of two or more correlated sources and characterizes lower bounds on the achievable rates. More specifically, it proves that when performing distributed coding (i.e. separate encoding and joint decoding), the same optimal rates can be achieved as when performing conventional coding (i.e. joint encoding and decoding), with a residual error probability which converges towards 0 for long sequences. The Wyner-Ziv theorem extends this result for lossy coding with Side Information (SI), under the assumptions that the sources are jointly Gaussian and a Mean Square Error (MSE) distortion measure is used.

The principle of distributed coding can also be applied to video coding. More specifically, Distributed Video Coding (DVC) relies on a statistical framework, unlike the deterministic approach of conventional MPEG and ITU-T coding schemes. Thanks to its properties, DVC offers a number of potential advantages and functionalities. Per consequent, DVC has gained a lot of interest over the last few years and has been the subject of numerous publications. Overviews can be found in [3][4][5].

Using DVC, the computational complexity can be flexibly distributed between the encoder and the decoder, and in particular, it allows encoding with very low complexity. However, the complexity advantage provided by DVC may be very transient, as with Moore's law, computing power increases exponentially and makes costeffective within a couple of years the implementation that is not manageable today. The absence of the prediction loop prevents drifts in the presence of transmission errors. Along with the built-in joint source-channel coding structure, it implies that DVC has improved error resilience. This property may prove appealing for video transmission over error-prone network channels. Given the absence of the prediction loop, DVC is also enabling codec independent scalability. Namely, a DVC enhancement layer can be used to improve a base layer without deterministically knowing it but rather relying only on some correlation knowledge. Camera sensor networks and multi-view coding is another domain where DVC shows promises. On top of the above benefits for mono-view, DVC allows for an architecture where cameras do not need to communicate, while still enabling the exploitation of inter-view correlation during joint decoding. This may prove an important architectural advantage, avoiding complex and power consuming networking.

Nowadays, state-of-the-art DVC coding schemes [6][7] consistently match or outperform H.264/AVC Intra, except for scenes with complex motion. For scenes with low motion, the gain can reach up to $5 \mathrm{~dB}$. When compared to 
H.264/AVC No Motion, the performance of DVC typically remains lower. However, DVC shows strong performance for scenes with simple and regular global motion, where it outperforms H.264/AVC No Motion due to the inability of the latter to efficiently exploit temporal redundancy. In terms of computational complexity, DVC schemes achieve significantly lower encoding complexity when compared to H.264/AVC Intra and H.264/AVC No Motion.

DVC is especially appealing for a new class of up-link applications such as low-power sensor networks, wireless video surveillance cameras and mobile communication devices. Eleven promising applications particularly well suited for DVC have been identified in [8]. While most of the initial interest in distributed coding principles has been towards video coding, it is now straightforward that other applications, such as media authentication, secure biometrics and tampering detection, may also benefit from these ideas.

Rapid technology evolution will soon enable the capture and display of video content with tremendously higher quality and resolutions. However, the progress in network infrastructure cannot keep pace with the dramatically increasing data rates and breakthrough in video compression is as needed as ever.

In this presentation, we offer a survey of recent trends and perspectives in DVC. More specifically, we discuss recent contributions addressing some open research issues such as coding efficiency, complexity, error resilience, scalability, multi-view coding, and applications beyond coding. We then identify challenges and hurdles ahead which need to be successfully tackled in order to bring DVC closer to market adoption and commercial exploitation. Finally, we assess the most promising avenues and outlook for future research directions in order to reach the breakthrough in video compression needed for the next generation of video products and services.

\section{REFERENCES}

[1] J. Slepian and J. Wolf, "Noiseless Coding of Correlated Information Sources", IEEE Trans. on Information Theory, vol. 19, no. 4, July 1973.

[2] A. Wyner and J. Ziv, "The Rate-Distortion Function for Source Coding with Side Information at the Decoder”, IEEE Trans. on Information Theory, vol. 22, no. 1, January 1976.

[3] C. Guillemot, F. Pereira, L. Torres, T. Ebrahimi, R. Leonardi and J. Ostermann, "Distributed Monoview and Multiview Video Coding”, IEEE Signal Processing Magazine, vol. 24, no. 5, 2007.

[4] P.L Dragotti and M. Gastpar, Distributed Source Coding: Theory, Algorithms and Applications, Academic Press, February 2009.

[5] F. Dufaux, W. Gao, S. Tubaro, A. Vetro, Distributed Video Coding: Trends and Perspectives, EURASIP Journal on Image and Video Processing, vol. 2009, Article ID 508167, doi:10.1155/2009/508167, 2009.
[6] X. Artigas, J. Ascenso, M. Dalai, S. Klomp, D. Kubasov, M Ouaret, "The Discover Codec: Architecture, Techniques and Evaluation”, in Proc. of Picture Coding Symposium (PCS), Lisboa, Portugal, November 2007.

[7] J. Ascenso, C. Brites, F. Dufaux, A. Fernando, T. Ebrahimi, F. Pereira and S. Tubaro, The VISNET II DVC Codec: Architecture, Tools and Performance, in Proc. 18th European Signal Processing Conference (EUSIPCO 2010), Aalborg, Denmark, August 2010.

[8] F. Pereira, L. Torres, C. Guillemot, T. Ebrahimi, R. Leonardi and S. Klomp, "Distributed Video Coding: Selecting the Most Promising Application Scenarios”, Signal Processing: Image Communication, Vol. 23, no. 5, pp. 339-352, June 2008. 